\title{
Surface Charge Polarization at the Interface: Enhancing the Oxygen Reduction via Precise Synthesis of Heterogeneous Ultrathin Pt/PtTe Nanowire
}

Hui-Hui Li,,$^{\dagger}{ }^{\nabla}$ Mao-Lin Xie, ${ }^{\dagger},{ }^{\nabla}$ Chun-Hua Cui $,{ }^{\dagger}, \nabla$ Da He, ${ }^{\dagger}$ Ming Gong, ${ }^{\S}$ Jun Jiang, ${ }^{+*}$ Ya-Rong Zheng, ${ }^{\dagger}$ Gang Chen, "Yong Lei" and Shu-Hong Yü*

†Division of Nanomaterials and Chemistry, Hefei National Laboratory for Physical Sciences at Microscale, Collaborative Innovation Center of Suzhou Nano Science and Technology, Department of Chemistry, University of Science and Technology of China, Hefei, Anhui 230026, P. R. China

Collaborative Innovation Center of Chemistry for Energy Materials, Hefei Science Center CAS, University of Science and Technology of China, Hefei, Anhui 230026, P. R. China.

${ }^{\S}$ Engineering and Materials Science Experiment Center, University of Science and Technology of China, Hefei, Anhui 230026, P. R. China.

"Shanghai Synchrotron Radiation Facility, Shanghai Institute of Applied Physics, Chinese Academy of Sciences, Shanghai 201204, P. R. China.

\#Ilmenau University of Technology, Institute of Physics and IMN MacroNano®(ZIK), Prof. Schmidt Str. 26, 98693 Ilmenau, Germany 
Chemicals. $\mathrm{Na}_{2} \mathrm{TeO}_{3}$, poly(vinyl pyrrolidone) (PVP, degree of polymerization: $360^{\circ}$ ), hydrazine hydrate $(85 \% \mathrm{w} / \mathrm{w} \%)$, aqueous ammonia solution $(25-28 \% \mathrm{w} / \mathrm{w} \%)$, ethanol, isopropyl alcohol and ethylene glycol (EG) were purchased from Shanghai Chemical Reagent Co. Ltd, and Nafion (5 wt.\%) from Sigma-Aldrich, and $\mathrm{K}_{2} \mathrm{PtCl}_{4}$ from $\mathrm{J} \& \mathrm{~K}$ chemical Ltd. All the chemical reagents used as received without further purification. Doubly distilled deionized water (DIW, 18.2 M $\Omega$ ) was used for all preparations in the synthesis of ultrathin Te NWs and Te-based NWs.

Instruments. X-ray powder diffraction (XRD) patterns were obtained from a Japan Rigaku DMax- $\gamma \mathrm{A}$ rotation anode X-ray diffractometer equipped with graphite monochromatized $\mathrm{Cu}-\mathrm{K}$ radiation $(\lambda=1.54178 \AA$ ). Scanning electron microscoe (SEM) images were taken with a Zeiss Supra 40 scanning eletron microscope at an acceleration voltage of $5 \mathrm{kV}$. Transmission electron microscope (TEM) and high-resolution transmission electron microscope (HRTEM) observations were performed on JEOL$2010 \mathrm{~F}$ with an acceleration voltage of $200 \mathrm{KV}$. Energy dispersive spectrometer (EDS) and scanning transmission electron microscopy energy dispersive spectrometer (STEM EDS) element mapping were carried out on Inca Oxford equipped on JEOL-2010F. Electrochemical measurements were performed on an electrochemical workstation (Autolab, Swiss). 

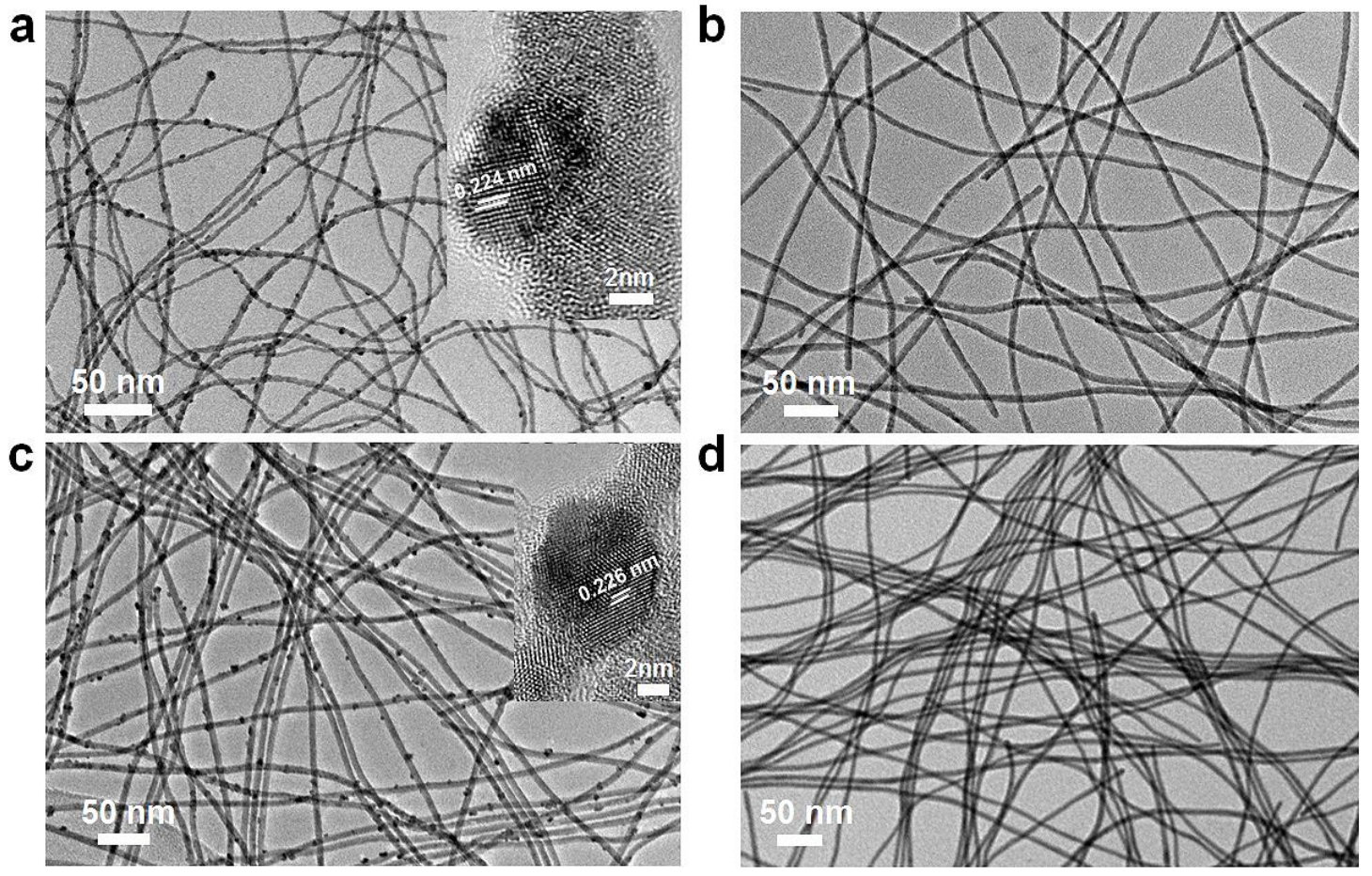

Figure S1. TEM and HRTEM (inset) images of the heterogeneous (a) and homogeneous (b) PtPdTe NWs using 0h-aged $\mathrm{K}_{2} \mathrm{PtCl}_{4}$ and $7 \mathrm{~h}$-aged $\mathrm{K}_{2} \mathrm{PtCl}_{4}$ mixed with $\mathrm{K}_{2} \mathrm{PdCl}_{4}$ solution as precursors, respectively. TEM and HRTEM (inset) images of the heterogeneous (c) and homogeneous (d) PtAuTe NWs using 0 h-aged $\mathrm{K}_{2} \mathrm{PtCl}_{4}$ and $7 \mathrm{~h}$-aged $\mathrm{K}_{2} \mathrm{PtCl}_{4}$ mixed with $\mathrm{HAuCl}_{4}$ solution as precursors, respectively.

Table S1. Local structure parameters around Pt estimated by EXAFS analysis.

\begin{tabular}{cccccc}
\hline Sample & shell & $\mathrm{N}$ & $\mathrm{R}(\AA)$ & $\sigma^{2}\left(10^{-3} \AA^{2}\right)$ & $\Delta \mathrm{E}_{0}(\mathrm{eV})$ \\
\hline \multirow{2}{*}{$\mathrm{EG}(7 \mathrm{~h})$} & $\mathrm{Pt}-\mathrm{Cl}$ & 3.1 & 2.32 & 2.5 & -1.3 \\
& $\mathrm{Pt}-\mathrm{Pt}$ & 1.3 & 2.83 & 10.5 & 4.5 \\
\hline
\end{tabular}

Here, $N, R, \sigma^{2}$ and $\Delta E_{0}$, represent the coordination number, bond distances, Debye-Waller factor and edge-energy shift, respectively. The error range of these parameters were estimated as $N \pm 4 \%, R \pm 1 \%$, $\sigma^{2} \pm 1 \%$ and $\Delta E_{0}, \pm 20 \%$. 


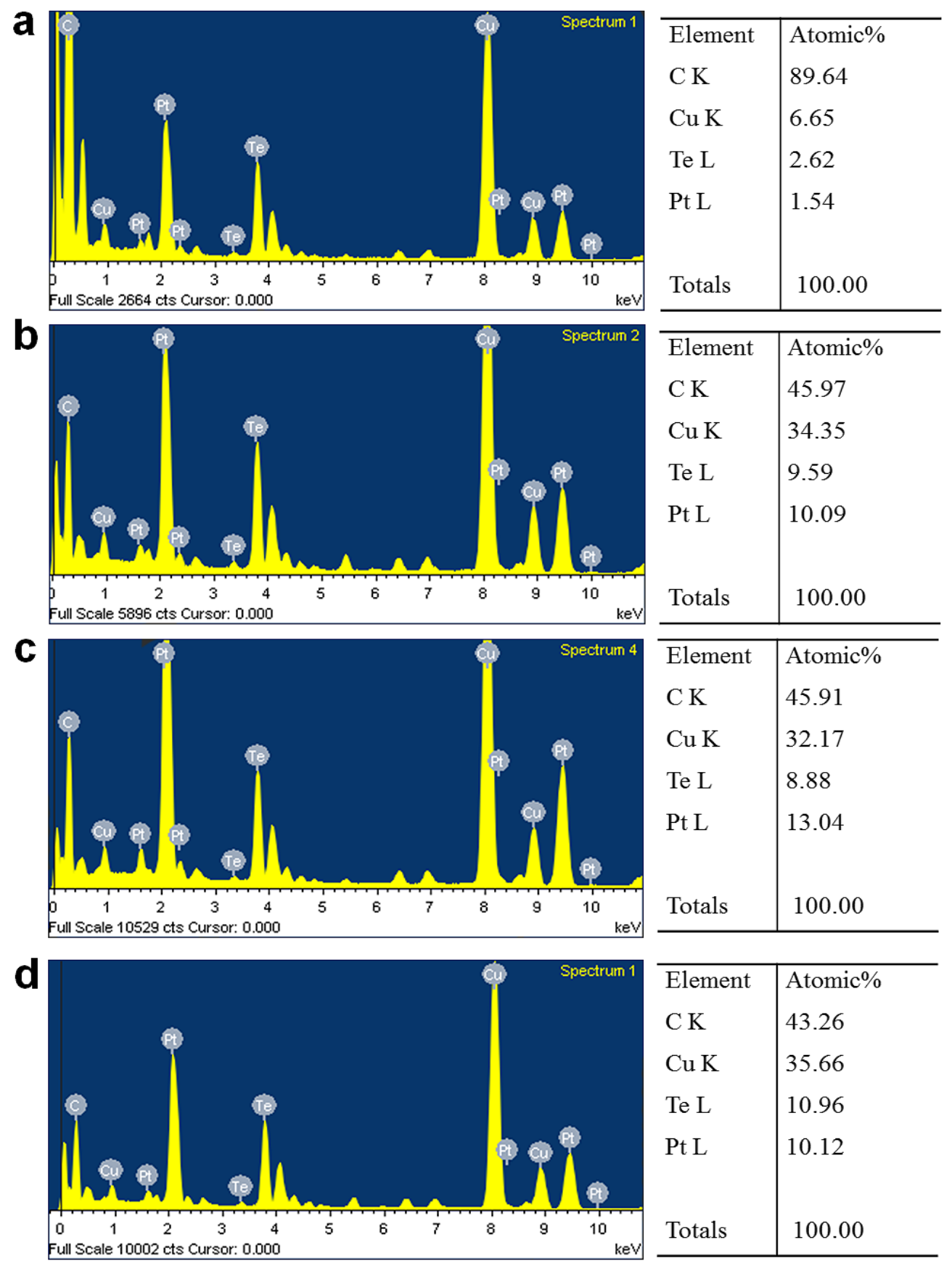

Figure S2. (a), (b), (c) and (d) are EDS analysis and atomic ratio of $\mathrm{Pt}$ and $\mathrm{Te}$ for $\mathrm{Pt} / \mathrm{Pt}_{37} \mathrm{Te}_{63}$, $\mathrm{Pt} / \mathrm{Pt}_{51} \mathrm{Te}_{49}, \mathrm{Pt} / \mathrm{Pt}_{60} \mathrm{Te}_{40}$ and $\mathrm{Pt}_{48} \mathrm{Te}_{52}$ catalysts, respectively. 

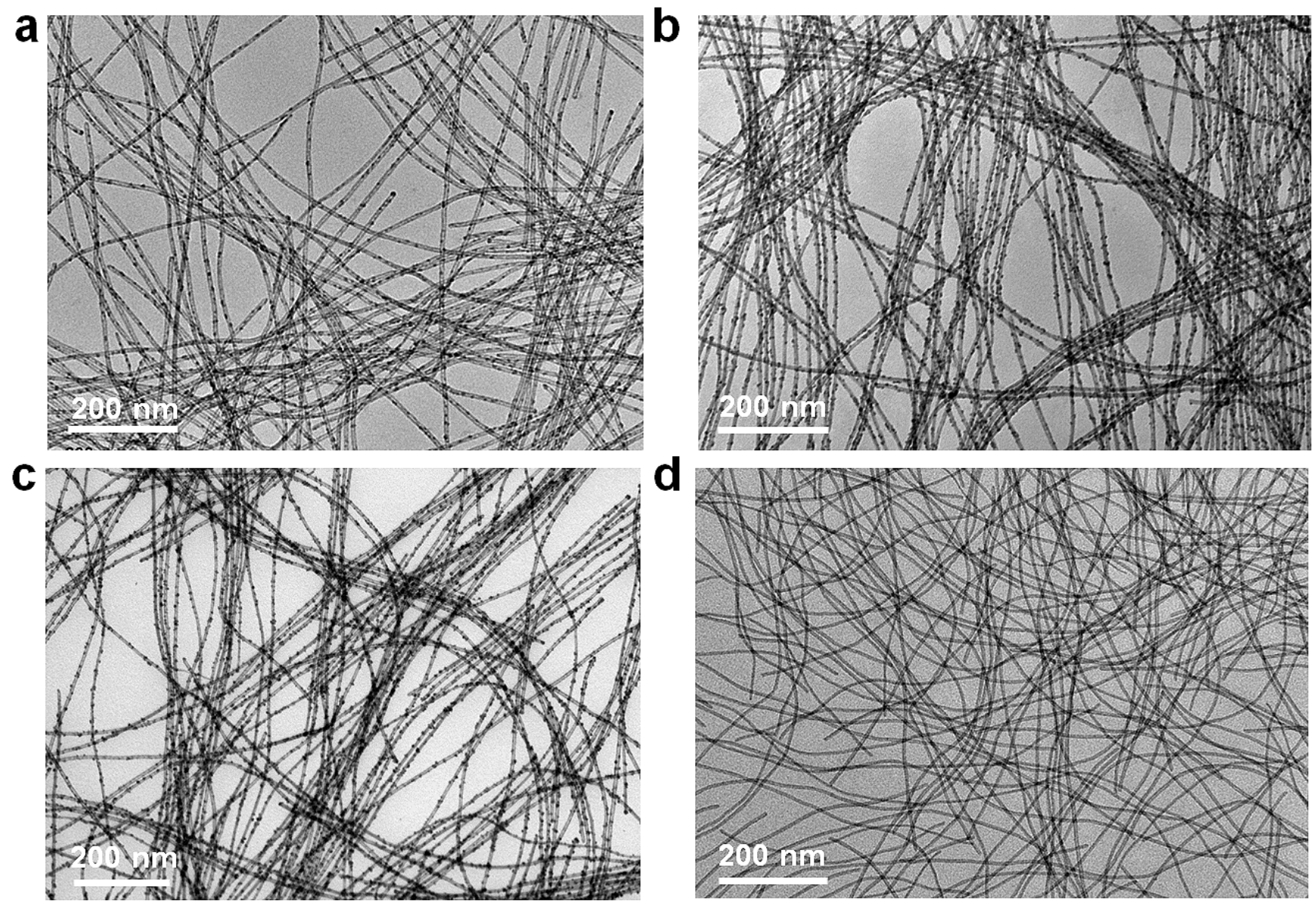

Figure S3. (a-d) TEM images of the $\mathrm{Pt} / \mathrm{Pt}_{37} \mathrm{Te}_{63}, \mathrm{Pt} / \mathrm{Pt}_{51} \mathrm{Te}_{49}, \mathrm{Pt} / \mathrm{Pt}_{60} \mathrm{Te}_{40}$ and $\mathrm{Pt}_{48} \mathrm{Te}_{52} \mathrm{NWs}$. 


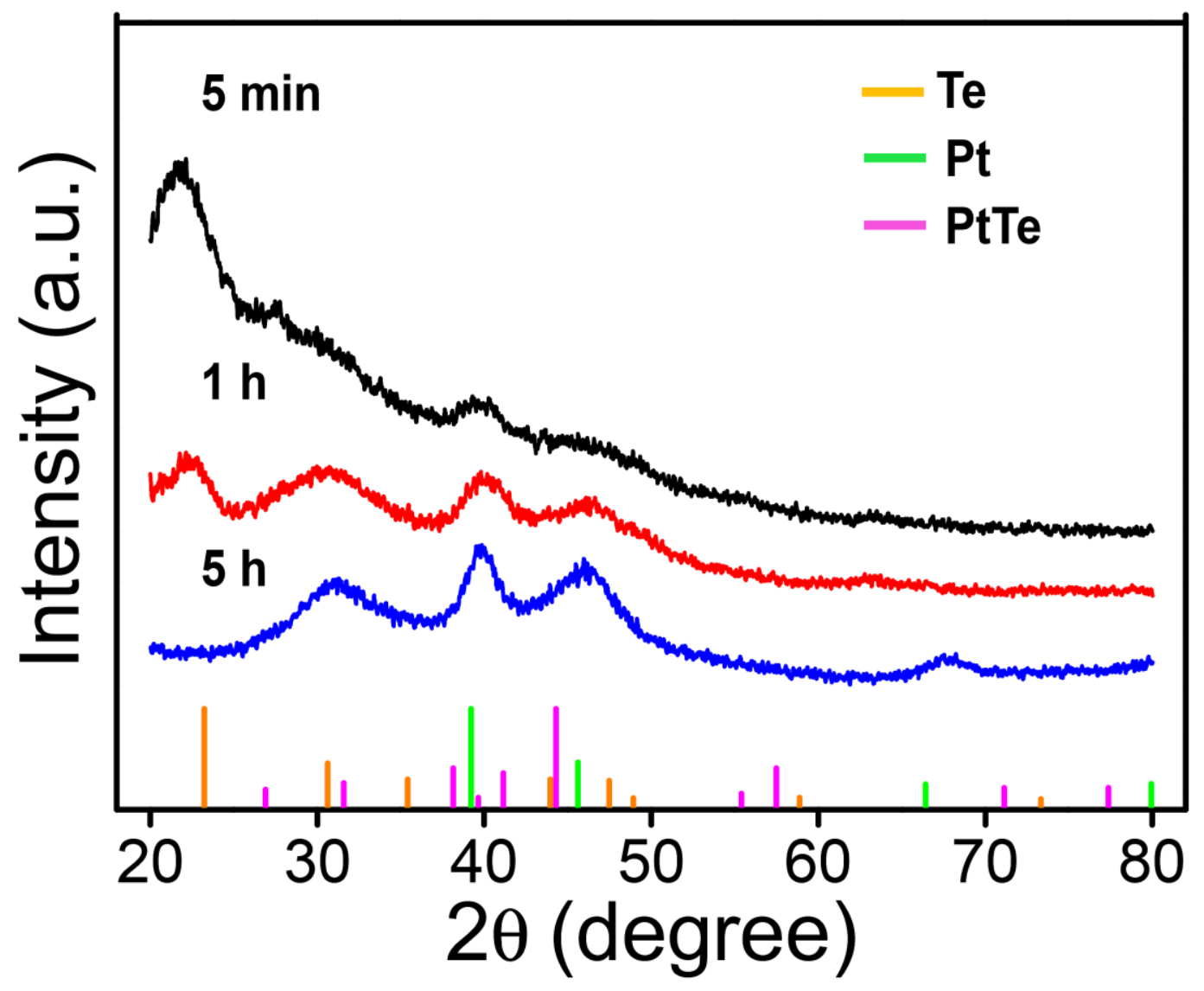

Figure S4. XRD pattern of Pt/tTe NWs after different reaction time to explore the formation of Pt NPs and PtTe NWs during the synthesis simultaneously. 

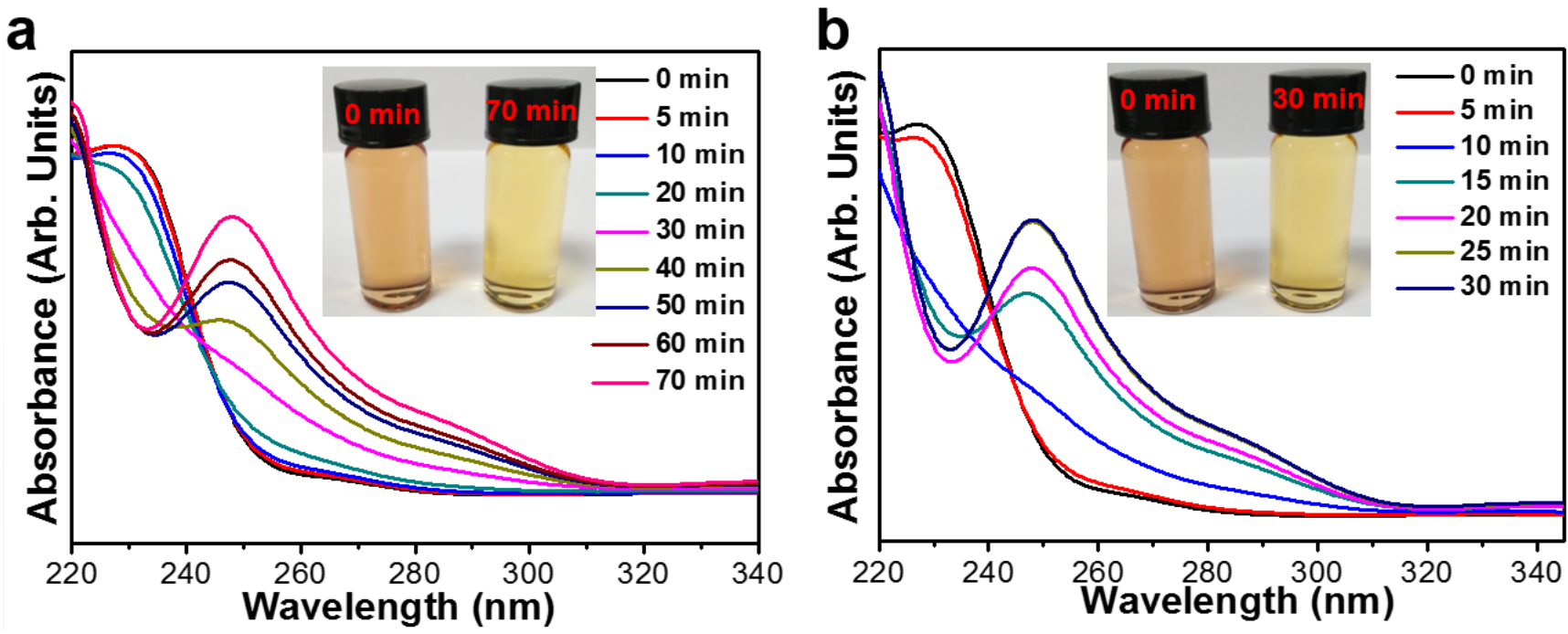

Figure S5. Temporal evolution of UV-vis absorption spectra of $\mathrm{K}_{2} \mathrm{PtCl}_{4}$ in $\mathrm{EG}$ solution aging at $50^{\circ} \mathrm{C}$ (a) and $80^{\circ} \mathrm{C}(\mathrm{b})$. The inset is the photos of $\mathrm{K}_{2} \mathrm{PtCl}_{4} \mathrm{EG}$ solution aging for $70 \mathrm{~min}$ at $50^{\circ} \mathrm{C}$ (a) and $30 \mathrm{~min}$ at $80^{\circ} \mathrm{C}(\mathrm{b})$, respectively.

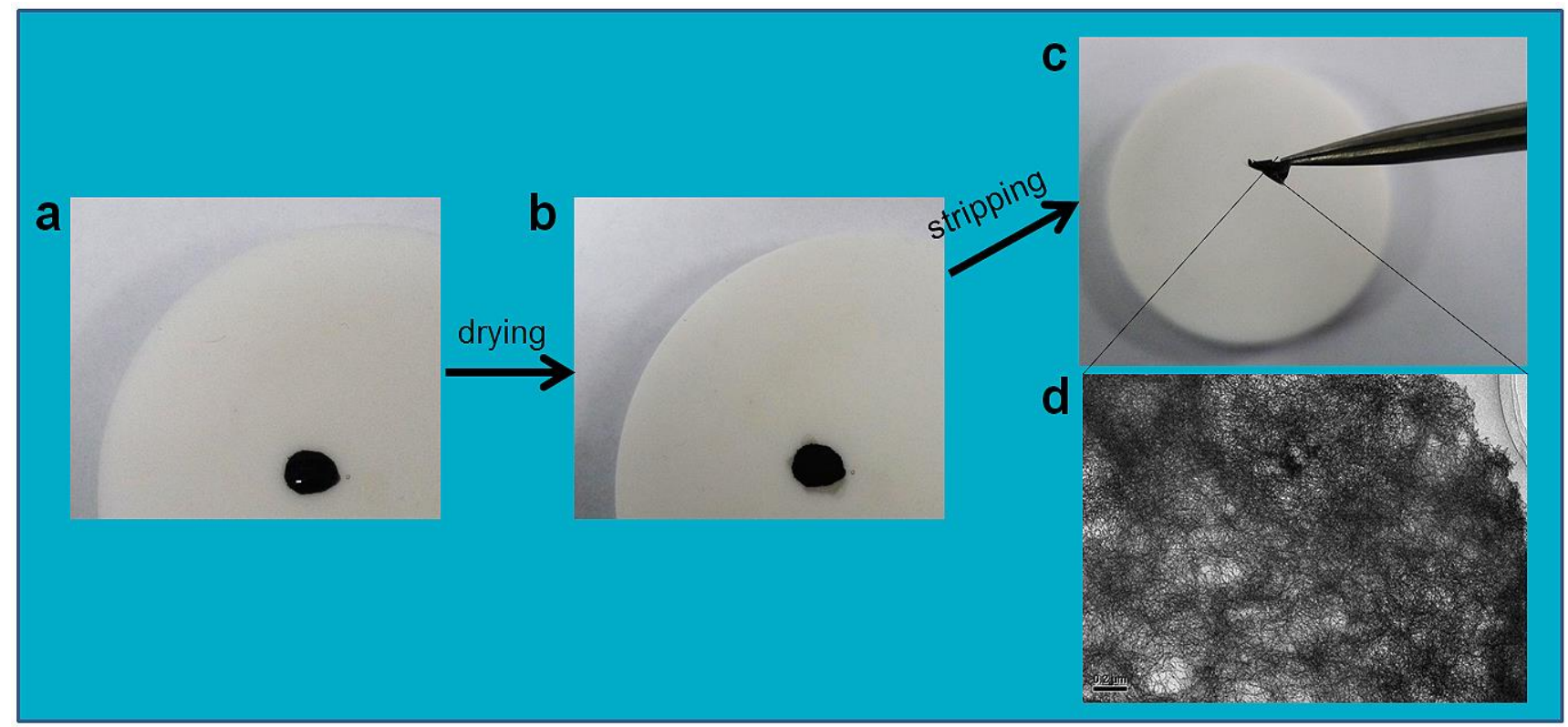

Figure S6. (a),(b) and (c) Photographs of a simple cast process of the formation of a the $\mathrm{Pt} / \mathrm{Pt}_{51} \mathrm{Te}_{49}$ catalysts film on a teflon substrate. When we add the catalysts suspension on a teflon substrate, the film forms after the ethanol evaporated completely. In the same way, a thin film will form when the NWs are added on the RDE prior to electrocatalysis tests; the film would facilitate the effective mass transport of reactants and products. (d) TEM images of film after sonication proving the network structure. 


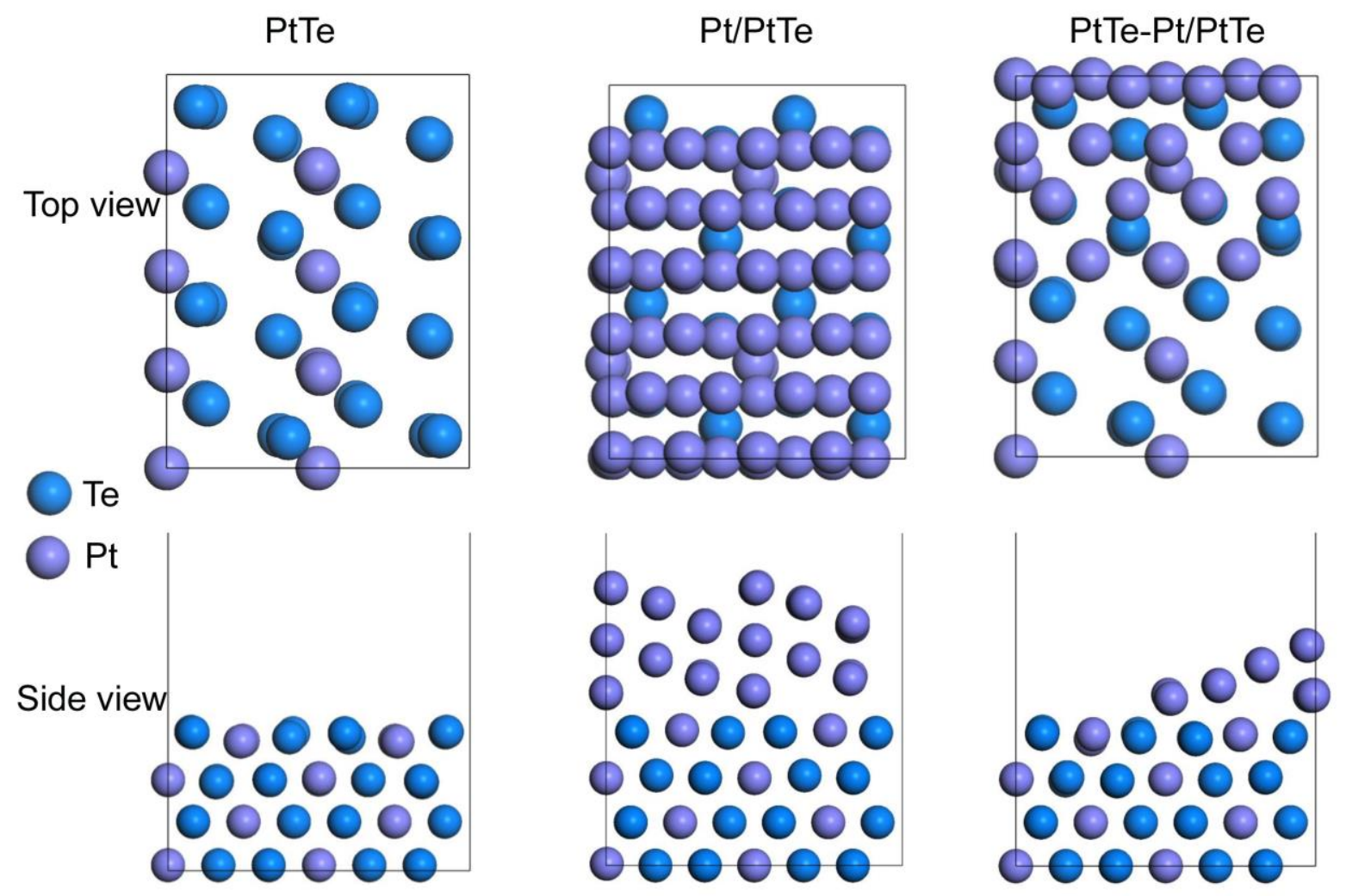

Figure S7. The atomic models from the top and side view for the PtTe surface, fully-covered Pt/PtTe surface, and half-covered PtTe-Pt/PtTe surface (the interfacing part between the exposed PtTe surface and Pt NP of the Pt/PtTe NW).

A system of $3 \sqrt{ } 2 \times 2 \sqrt{ } 2$ supercell with 4 layers of PtTe (110) were constructed to model the PtTe surface, based on which 3 layers of Pt (112) were added to represent the fully-covered Pt/PtTe surface. As for the half-covered PtTe-Pt/PtTe interface, a Pt cluster was built over PtTe(110). In all of the geometry optimization calculations, two bottom layers were fixed while other atoms are fully relaxed. Facet tests were done which confirmed the match of Pt (112) facet with Te (110). PtTe (110) were chosen to be interfaced with Pt (112).

An energy cutoff of $400 \mathrm{eV}$ was used for the plane-wave expansion of the electronic wave function. The force and energy convergence criterion was set to $0.01 \mathrm{eV} / \AA$ and $10^{-5} \mathrm{eV}$, respectively. The Brillouin zone was integrated by using Monkhorst-Pack generated sets of k-points. The $7 \times 7 \times 7$ k-point mesh was chosen for bulk computations while $3 \times 3 \times 1 \mathrm{k}$-point tests were done for the surfaces. 

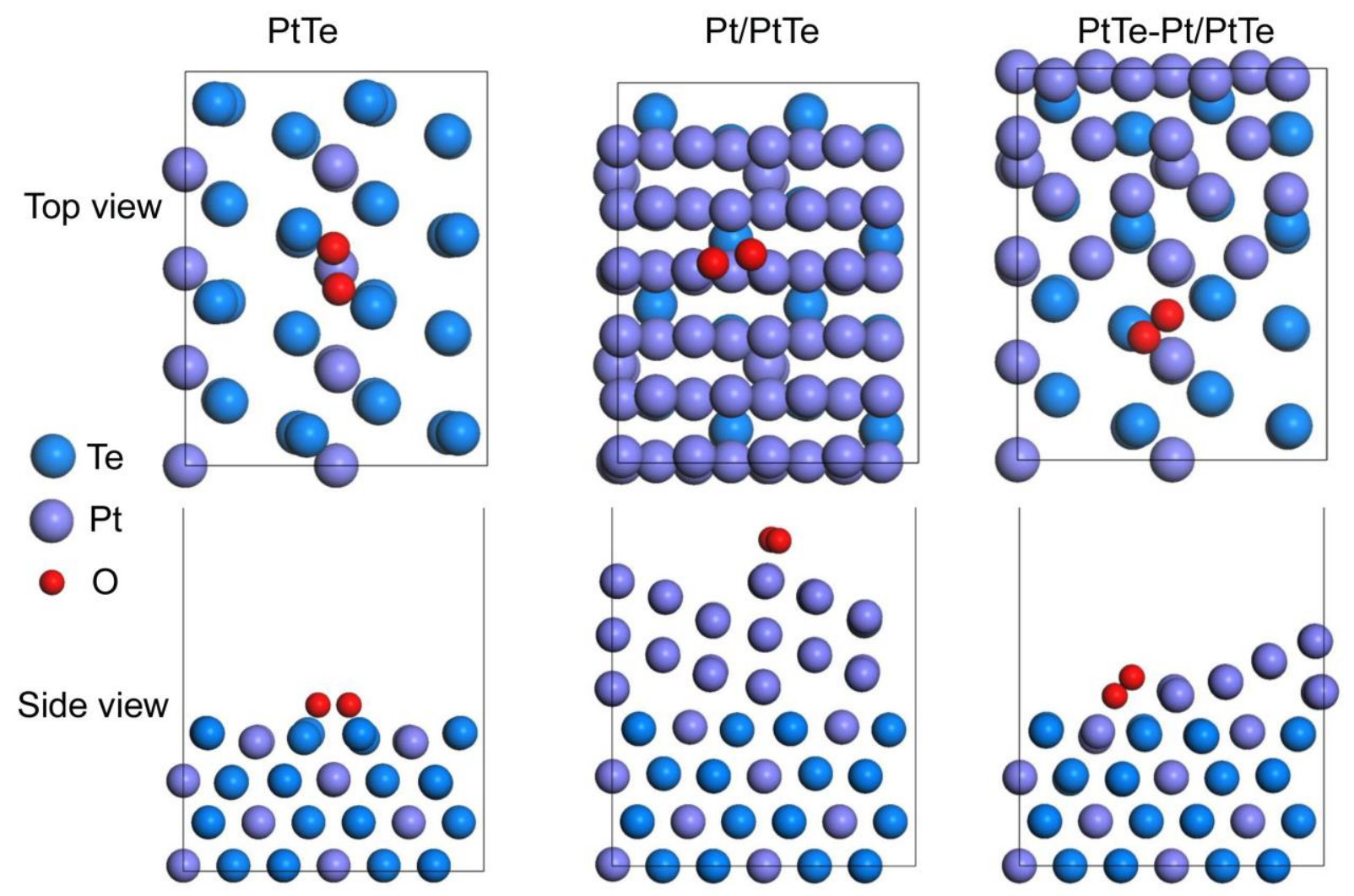

Figure S8. The simulated adsorption of $\mathrm{O}_{2}$ on PtTe, Pt/PtTe, PtTe-Pt/PtTe surface.

An $\mathrm{O}_{2}$ molecule is adsorbed to PtTe or Pt/PtTe by two metal atoms in parallel to the surface, while it straddles over the PtTe and Pt/PtTe parts at the PtTe-Pt/PtTe interface, in which each $\mathrm{O}$ atom attached to one Pt from those two parts separately. Their O-O bond lengths are not sensitive to the surface situation, varying from 1.46 to 1.42 Angstrom. Similarly, the differences between the O-metal atom distances are very small for these three models. On the other hand, the adsorption energy varies greatly in the three model systems, as displayed in table $\mathrm{S} 1$. The PtTe-Pt/PtTe interface is expected to more efficient in adsorb $\mathrm{O}_{2}$ molecule so that enhance $\mathrm{ORR}$ reaction rate. 

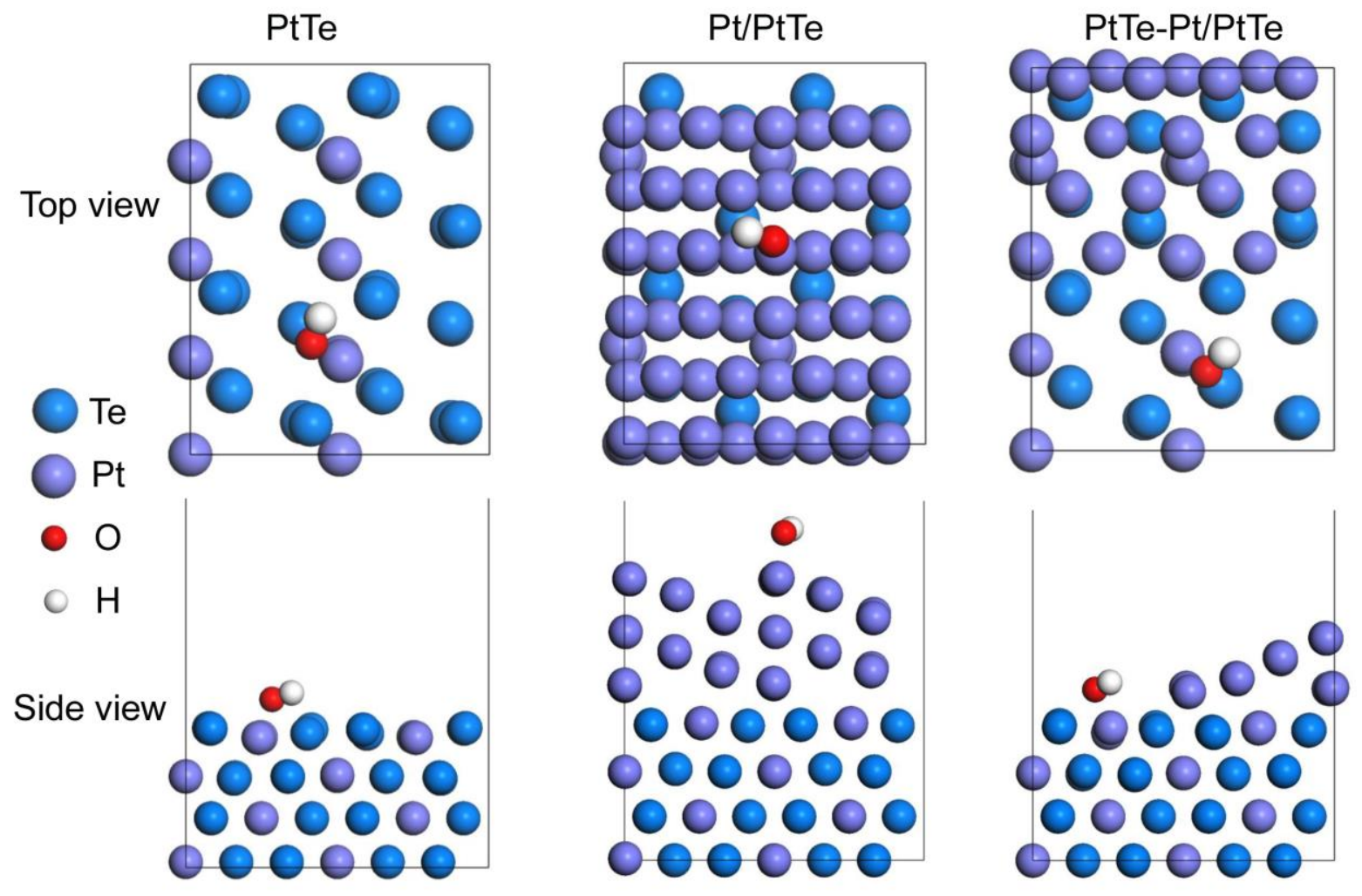

Figure S9. The simulated adsorption of $-(\mathrm{OH})$ with Pt sites on PtTe, Pt/PtTe, PtTe-Pt/PtTe surface.

The $-(\mathrm{OH})$ group is adsorbed to the PtTe, Pt/PtTe, PtTe-Pt/PtTe surface through one Pt atom. The Pt- $(\mathrm{OH})$ bond lengths of the three systems are similar, varying from 2.04 to 1.96 Angstrom. Different adsorption energies as listed in Table S1 imply that PtTe-Pt/PtTe might have the lowest energy barrier for breaking the Pt- $(\mathrm{OH})$ bond. 

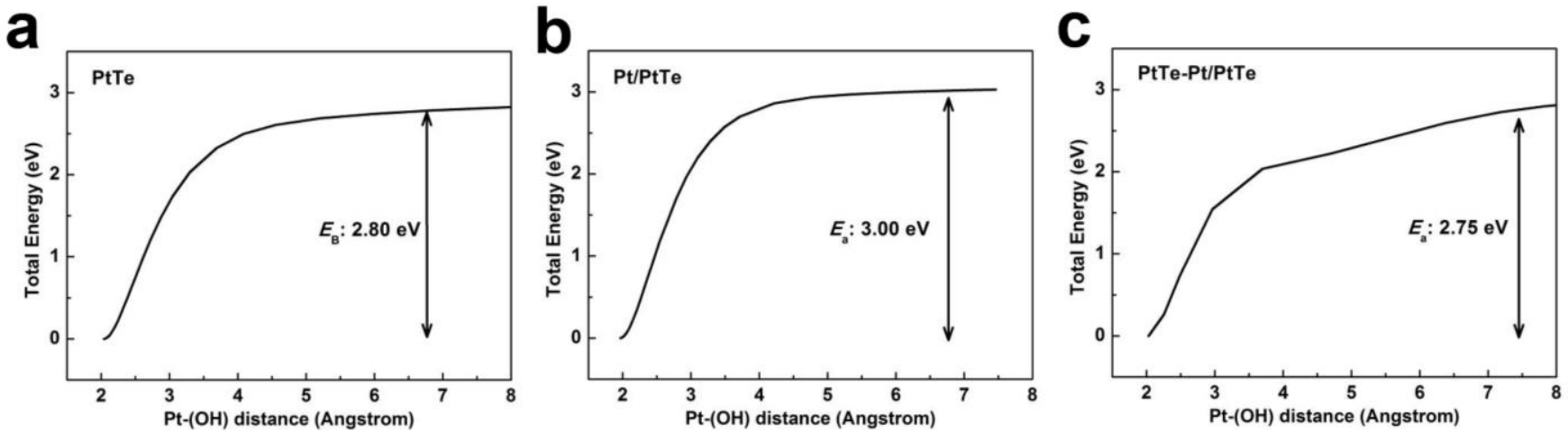

Figure S10. The computed energy potential surfaces along the breaking of Pt- $(\mathrm{OH})$ bonds on PtTe, $\mathrm{Pt} / \mathrm{PtTe}, \mathrm{PtTe}-\mathrm{Pt} / \mathrm{PtTe}$ surface as the function of $\mathrm{Pt}-(\mathrm{OH})$ distance. The activation energies were extracted from the saturated points.
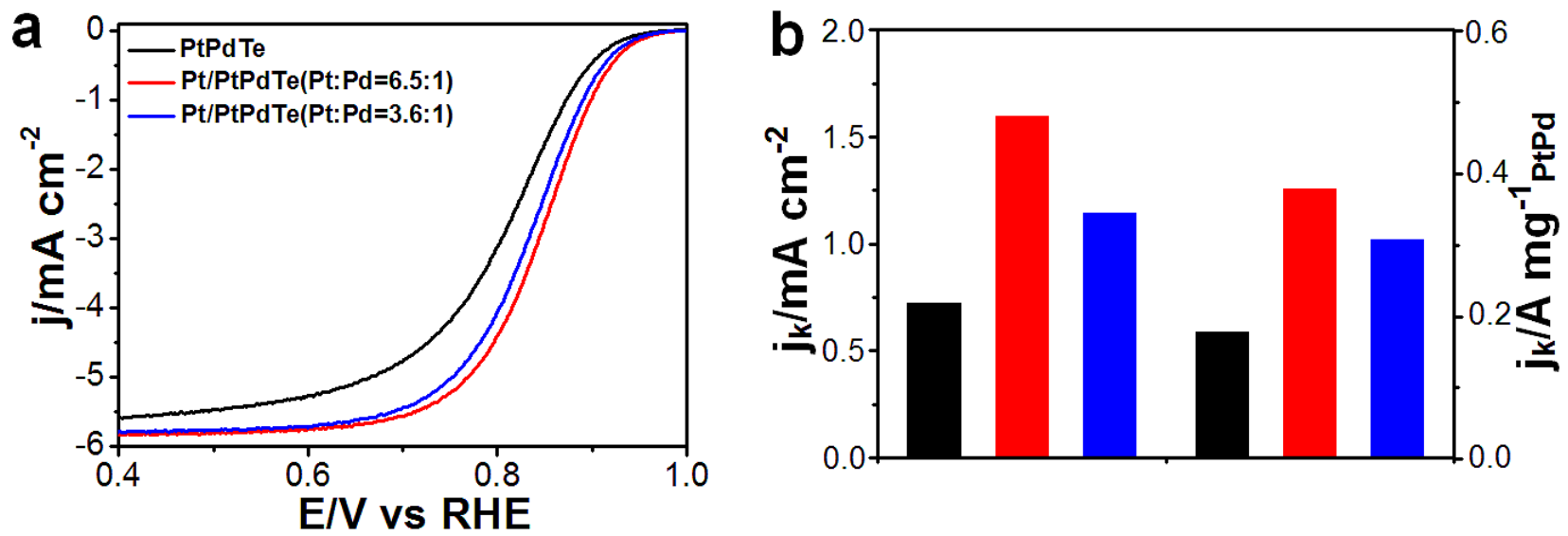

Figure S11. (a) ORR polarization curves for the homogeneous and heterogeneous PtPdTe catalysts in $\mathrm{O}_{2}$-saturated $0.1 \mathrm{M} \mathrm{HClO}_{4}$ solution with a sweep rate of $5 \mathrm{mV} \mathrm{s}^{-1}$ and a rotation rate of $1600 \mathrm{rpm}$. (b) Specific activity and mass activity are depicted as kinetic-current densities $\left(j_{k}\right)$ normalized to the ECSA and the loading mass of the metal, respectively. The colour scheme in (a) applies to (b). 

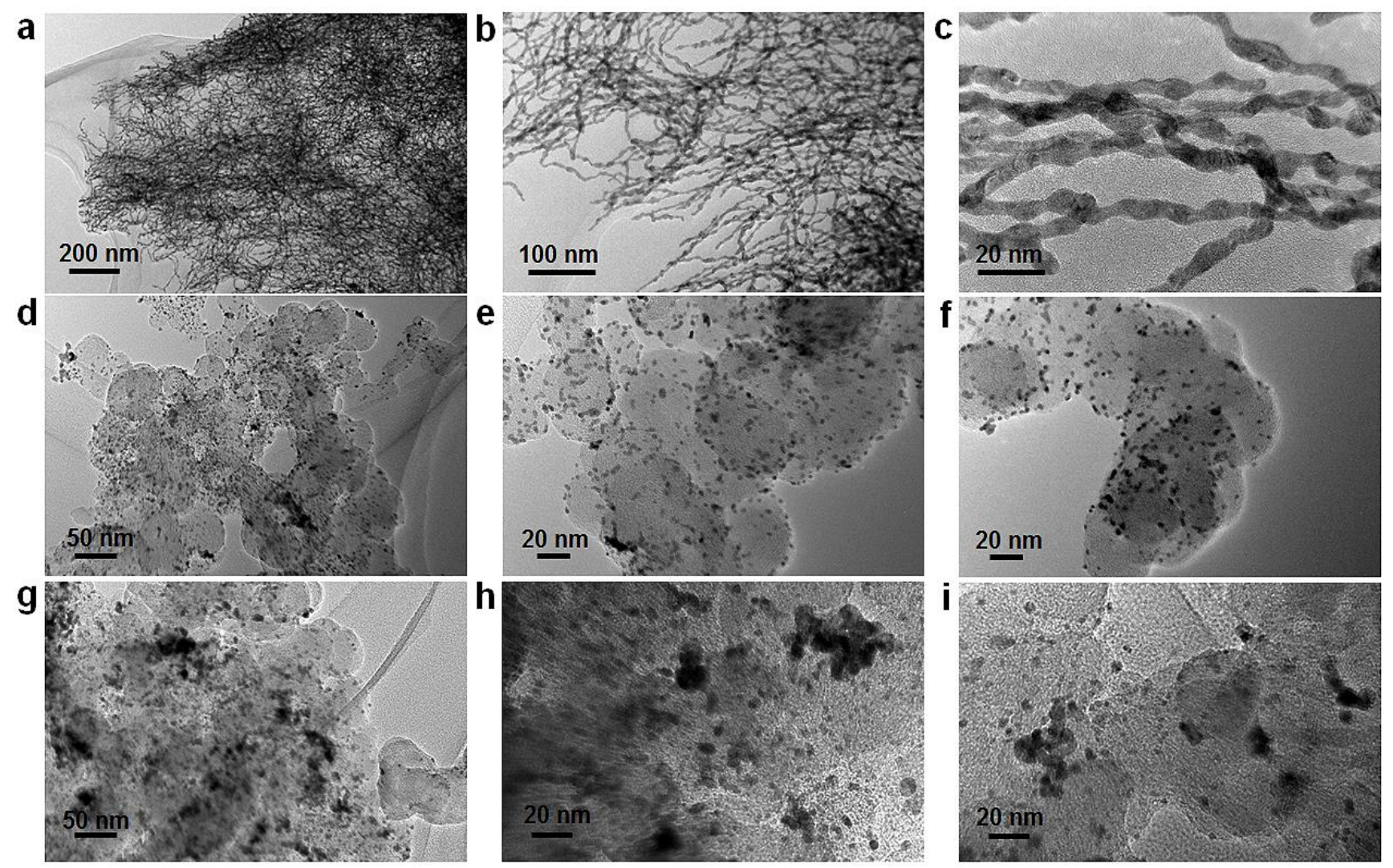

Figure S12. (a), (b) and (c) TEM images of the $\mathrm{Pt} / \mathrm{Pt}_{51} \mathrm{Te}_{49}$ catalysts after accelerated stability tests. (d), (e) and (f) TEM images of the Pt/C catalysts before the electrochemical activity tests. (g), (h) and (i) TEM images of the Pt/C catalysts after accelerated stability tests. 